\title{
Prevalence of internal carotid artery morphological variations and its association with cerebrovascular ischemic stroke (hospital-based study)
}

Noha AboElfetouh', Eman Khedr', Hesham Aboloyoun ${ }^{2}$, Mahmoud Nageeb $^{1 *}$ and Ahmed Nasreldein ${ }^{1}$

\begin{abstract}
Background and objectives: Morphological variations (MV) in the course of the internal carotid artery (ICA) are commonly identified. Their prevalence and relationship with ischemic stroke (IS) remain unclear. The present study attempted to estimate the prevalence and pattern of MV of ICA among Egyptian patients and to detect whether these MV associated with increased risk of IS or not.

Methods: Out of the recruited 550 acute ischemic stroke patients, 102 met the inclusion and exclusion criteria and were included in our study. Each patient was subjected to detailed history including risk factors and clinical assessment using the National Institute of Health Stroke Scale, mRS, and extracranial duplex ultrasound (US) assessment.

Results: Eleven patients out of 102 (10.8\%) showed MV in their ICA course, 6 of them were women and 5 were men. These MV were unilateral in 90.9\% (10/11) of cases and more in the left ICA (72.7\%), while one case (9.1\%) showed MV of both ICA. The commonest pattern of MV was tortuosity in 7 cases (63.6\%) and kinking in 4 cases (36.4\%). The history of previous IS and TIA was recorded in 9.1\% of patients with MV in ICA for each group, while the history of IS was recorded in 13.2\% of patients without MV and TIA in 4.3\% patients without MV in ICA. There were no statistically significant differences between both groups.
\end{abstract}

Conclusion: The prevalence of MV in the present study is 10.8\%. MV are more frequent in women and older patients and are not associated with an increased risk of ischemic stroke.

Keywords: Pattern, Morphologic, Variations, ICA, Ischemic, Stroke, Duplex

\section{Introduction}

In Egypt, there is a high crude prevalence rate of ischemic stroke (IS): 936/100,000 and 922/100,000 in Assiut and Qena governorates, respectively [1, 2], compared to prevalence rates in other Arabic counties [3]. Morphological variations (MV) of the internal carotid artery (ICA) are often classified into tortuosity, kinking, and coiling [4]. According to the previous

\footnotetext{
* Correspondence: ma7moudnageeb@yahoo.com

${ }^{1}$ Neurology Department, Assuit University, Assiut 71526, Egypt

Full list of author information is available at the end of the article
}

classification, tortuosity is an S- or C-shaped elongation or undulation in the course of ICA. Coiling was defined as elongation or redundancy of the ICA resulting in an exaggerated S-shaped curve or in a circular configuration. Kinking, the most frequently reported type of carotid abnormalities (CA), was described as an acute angulation of ICA and classified according to the severity of the angle between the two segments forming the kink [5]. The etiology of ICA MV can be either congenital or acquired. Congenital variations are observed in both youth adults and children. Acquired could be due to atheroma, 
aging, and/or hypertension [6]. The clinical significance of MV in ICA is not well studied; however, a tortious artery may increase the probability of vessel spasm [6], and some authors postulated that MV are associated with a higher risk of IS [7]. However, Radak et al. stated that IS accident occurs in about $11-33 \%$ of these cases [8]. The prevalence of ICA MV in IS patients ranged from 4 to $66 \%[7,9-11]$.

Despite their common occurrence, conclusive evidence about their prevalence and relationship with the IS is still lacking [6].

Up to our knowledge, the prevalence of ICA MV, their characteristics, and its impact on IS are not well studied among Egyptian patients. The aim of the study was to estimate the prevalence and pattern of morphological variations of the ICA among IS patients and to study if there is a link between these MV and the risk of IS occurrence or not.

\section{Main text}

\section{Patients and methods}

Our hospital-based study was conducted in the Neurology Department, Assuit University hospitals, during the period from May 1, 2018, to April 30, 2019.

One hundred two patients out of 550 recruited patients diagnosed with acute ischemic stroke (Fig. 1) fulfilled the following inclusion criteria: age $>18$ years, acute ischemic stroke, and transient ischemic attack in anterior circulation presented within $48 \mathrm{~h}$ diagnosed according to WHO diagnostic criteria. Exclusion criteria were patients with carotid stenosis due to atherosclerosis or dissection; patients with known vasculitis or discovered to have after duplex examination; patients with IHD, AF, and cardiomyopathy; patients hemodynamically unstable; patients difficult to be examined with duplex ultrasound; and refusal to participate.

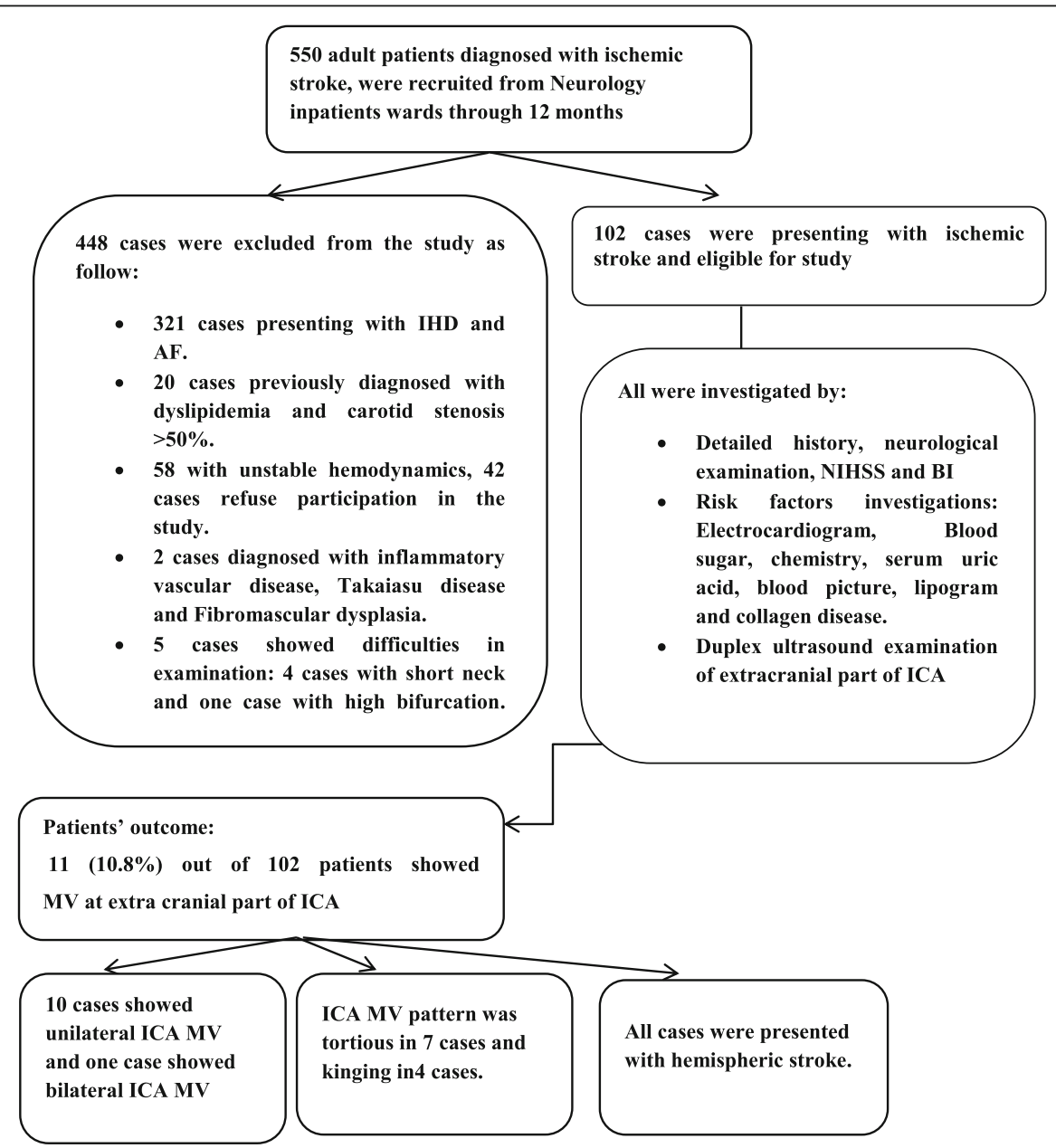

MV: morphological variations, ICA: internal carotid artery, IHD: ischemic heart disease,

NIHSS: national institute of health stroke scale, BI: barthel index scale.

Fig. 1 Flow chart 
All patients underwent full detailed history, general, and neurologic examination, and their stroke severity was assessed on admission using the National Institutes of Health Stroke Scale (NIHSS) and modified Rankin scale (mRS). All patients had computerized tomography (CT brain) using non-enhanced thin sections helical 16 multidetector CT (GE optima CT scanners made in Chicago, USA), and some patients with normal CT brain had additional magnetic resonance imaging (MRI) of the brain to confirm the diagnosis of stroke using a 1.5- $\mathrm{T}$ scanner (Achieva, Philips made in Amsterdam, Netherlands). Routine laboratory investigations and collagen profile screening were performed for the detection of IS risk factors. ECG and Echo were done to all patients to exclude the cardioembolic source of stroke.

Duplex ultrasound examination took place in the Neurovascular Ultrasound Laboratory at the Neurology Department in cooperation with ultrasound outpatients' clinic at the Department of Vascular and Endovascular Surgery, Assiut University Hospitals. All measurements were done using a high-resolution color duplex Philips EnVisor C and Philips HD5 (Philips Medical Systems, Nederland B.V.) with L $3-12 \mathrm{MHz}$ linear transducer probe. The examination was performed by two experienced sonographers. The extracranial vessels were examined using an examination protocol, and atherosclerotic changes were interpreted according to the criteria published by the Society of Radiologists in Ultrasound [12].

In our study, we defined abnormal shape and course of the extracranial vessels according to the modified Weibel and Fields classification [13]. The angle of the kink is measured between 2 lines parallel to the vessel's wall proximal and distal to the most extreme angulation presented in. The kink was considered mild if the angle is more than $60^{\circ}$ and moderate when the angle is between 30 and $60^{\circ}$. The kink is considered severe when the angle is less than $30^{\circ}$.

\section{Statistical analysis}

The data were analyzed using SPSS 16.0 software. Qualitative data were described in frequency using percentage (\%). Continuous variables were expressed in mean \pm SD. Comparative statistical analysis between variables of both groups, patients with versus without MV of ICA, was done using the chi-square for qualitative data and independent $t$ test for continuous variables using nonparametric statistical tests, Mann-Whitney test. A value of $P<0.05$ was considered statistically significant.

\section{Results}

Eleven patients with ischemic stroke showed MV in the ICA course at the extracranial part, proximal to the bifurcation of CCA on screening, and giving a relative frequency of MV in the ICA course $10.8 \%$.
The mean age of these patients with MV in the ICA course was $68.91 \pm 7.3$ years old and ranged from 60 to 80 years old, suggesting that the observed MV were acquired in etiology. Six of them were females $(54.5 \%)$ and 5 were males $(45.5 \%)$ (Table 1$)$.

All patients with MV had medical risk factors for IS in addition to MV. Nine of them $(81.8 \%)$ had combined DM and hypertension, and two cases (18.2\%) had either DM or hypertension. Ten cases (90.9\%) with ICA MV showed ICA stenosis $<50 \%$ and one of them showed ICA stenosis $<50 \%$ bilaterally, while no ICA stenosis was observed in one case (9.1\%). ICA stenosis $\geq 50 \%$ was not recorded in any case.

The pattern of ICA MV was tortious in 7 cases (63.6\%) and kinking in 4 cases (36.4\%). These MV were recorded unilateral in majority $(90.9 \%)$ of the cases and bilateral in one case only $(9.1 \%)$. Our data revealed that the MV predominated in the left ICA (72.7\%) than in the right (27.3\%) (the clinical data of patients with MV in ICA are illustrated in Table 2).

The clinical rating scales of these patients for neurological deficit and functional state assessment showed that the mean NIHSS score was $15.2 \pm 4.4$ ranging from 2 to 22 and mRS was $4 \pm 1.1$. We found no significant difference between the scores of NIHSS and mRS in both groups, $P>0.05$ (see Table 3). The history of previous IS and TIA was recorded in $9.1 \%$ of patients with MV in ICA for each group, while the history of IS was recorded in $13.2 \%$ of patients without MV and TIA in $4.3 \%$ patients without MV in ICA. There were no statistically significant differences between both groups, $P$ $>0.05$.

Neuroimaging of patients with ICA MV showed multiple lacunar infarction in $54.5 \%(n=6$ cases), massive infarction in $27.3 \%$ ( $n=3$ cases), and White matter ischemia in $18.2 \%$ ( $n=2$ cases). Patients' individual data are illustrated in Table 2. A comparison between demographic data, clinical presentation, risk factors for CVS, and history of previous cerebrovascular insult as well as TIA preceding the current insult among patients with versus without MV in the ICA course shows no significant differences between groups in any of these items (Table 3).

\section{Discussion}

This study aimed to estimate the prevalence of MV in the ICA course among ischemic stroke patients and it was $10.8 \%$. Tortuosity was the commonest MV observed in our patients (63.6\%), followed by kinking (36.4\%). There were no differences in the liability to IS or TIA among patients with MV and without, and the stroke severity among both groups was not affected by the presence of the MV. 
Table 1 Demographic and clinical data of ischemic stroke patients with and without internal carotid artery morphological variations

\begin{tabular}{|c|c|c|c|}
\hline & Patients with MV in the ICA course $(n=11)$ & Patients without MV in the ICA course $(n=91)$ & $P$ value \\
\hline$<60$ years & $0(0 \%)$ & $23(25.3 \%)$ & 0.075 \\
\hline$\geq 60$ years & $11(100 \%)$ & $78(85.7 \%)$ & \\
\hline Sex & & & 0.653 \\
\hline Male & $5(45.4 \%)$ & $35(38.5 \%)$ & \\
\hline Female & $6(54.6 \%)$ & $56(61.5 \%)$ & \\
\hline Clinical presentation & & & 0.631 \\
\hline Right-sided hemiplegia or hemiparesis & $7(63.6 \%)$ & $51(56 \%)$ & \\
\hline Left-sided hemiplegia or hemiparesis & $4(36.4 \%)$ & $40(44 \%)$ & \\
\hline Risk factor & & & 0.179 \\
\hline Negative risk factor & $0(0 \%)$ & $13(14.4 \%)$ & \\
\hline Positive risk factors & $100 \%$ & $78(85.7 \%)$ & \\
\hline One risk factor (D.M. or HTN) & $2(18.2 \%)$ & $19(20.8 \%)$ & \\
\hline Two risk factors & 9 (81.8\%) & 59 (64.8\%) & \\
\hline
\end{tabular}

D.M. diabetes mellitus, HTN hypertension, MV morphological variations, ICA internal carotid artery

Various studies [14-16] have found that ICA MV was associated with ischemic stroke. Cerebral ischemia from ICA MV occurs through two mechanisms: a thromboembolic mechanism from endothelial lesions due to changes in the local flow at the site of arterial bending, and/or a hemodynamic mechanism (hemodynamic stroke) that plays an important role under both neutral and dynamic conditions.

Sacco and colleagues [17] used carotid ultrasound to study ICA MV among 1217 patients, $12.7 \%$ only of them had IS or TIA. They found that the prevalence of MV among the whole population was $26.2 \%$ which is much higher than our prevalence. This could be attributed to the difference in the sample size, selection criteria of the study population, and ethnicity. They did not find any difference between sexes under the age of 60 , while above the age of 60 , the prevalence of MV was higher in women than in men. Like our results, we found an insignificant higher prevalence rate among females. A more prominent change in vessel wall morphology among females could be linked to hormonal processes especially among postmenopausal women [7]. The tortuosity and kinking were the commonest ICA MV reported by them, and they were more frequently found on the left side which was in concordance to our results. Like our results, they did not show any relationship between ICA MV with IS or TIA.

Togay-Isikay et al. [13] studied 345 consecutive patients using carotid ultrasound, 102 patients out of them had stroke and TIA. They found that the prevalence of MV among all study population was $24.6 \%$, and among patients with a history of IS or TIA, it was $15.7 \%$ which is slightly higher than ours. Kinking followed by tortuosity was the most common MV encountered by them

Table 2 Individual data for patients with internal carotid artery morphological variations

\begin{tabular}{lllllll}
\hline Clinical presentation & Age & Sex & Risk factor & NIHSS & Rt ICA & Lt ICA \\
\hline Lt hemiplegia & 75 & Male & D.M., HTN & 22 & Occluded & Tortuous with stenosis $<50 \%$ \\
Rt hemiparesis & 60 & Female & D.M., HTN & 10 & Normal & Tortuous with stenosis $<50 \%$ \\
Improved RT hemiparesis and dysarthria & 65 & Male & HTN & 4 & Normal & Mod kink stenosis $<50 \%$ \\
Rt hemiplegia & 65 & Male & D.M. & 14 & Normal & Mild kink stenosis $<50 \%$ \\
Triple stroke recent Rt & 80 & Male & D.M., HTN & 18 & Normal & Tortuous with stenosis $<50 \%$ \\
Lt hemiplegia & 60 & Female & D.M., HTN & 18 & Tortuous with stenosis $<50 \%$ & Tortuous with stenosis $<50 \%$ \\
Rt hemiplegia & 80 & Female & D.M., HTN & 20 & Normal & Mild kink stenosis $<50 \%$ \\
Rt hemiplegia & 66 & Female & D.M., HTN & 14 & Mild kink with a normal lumen & Normal \\
Rt hemiparesis & 75 & Female & D.M., HTN & 8 & Tortuous with stenosis $<50 \%$ & Normal with stenosis $<50 \%$ \\
Rt hemiplegia & 67 & Male & D.M., HTN & 16 & Normal & Tortuous with stenosis $<50 \%$ \\
Lt hemiplegia & 65 & Female & D.M., HTN & 12 & Normal & Tortuous with stenosis $<50 \%$
\end{tabular}

NIHSS, Barthel, is a clinical rating scale for stroke. Lt left side, $R t$ right side, D.M. diabetes mellitus, HTN hypertension, ICA internal carotid artery, DCL disturbed conscious level 
Table 3 Demographic and clinical data of ischemic stroke patients with and without internal carotid artery morphological variations

\begin{tabular}{|c|c|c|c|}
\hline & $\begin{array}{l}\text { Patients with MV in the ICA course }(n= \\
\text { 11) }\end{array}$ & $\begin{array}{l}\text { Patients without MV in the ICA course }(n= \\
\text { 91) }\end{array}$ & $\begin{array}{l}P \text { value using the chi-square or } T \\
\text { test }\end{array}$ \\
\hline \multicolumn{4}{|c|}{ History of cerebrovascular insult } \\
\hline$\cdot$ TIA & $1(9.1 \%)$ & $4(4.3 \%)$ & 0.495 \\
\hline$\cdot$ CVS & $1(9.1 \%)$ & $13(14.3 \%)$ & 0.739 \\
\hline \multicolumn{4}{|c|}{ Neuroimaging findings } \\
\hline Normal CT brain & $0(0)$ & $5(5.2 \%)$ & 0.065 \\
\hline Lacunar infarctions & $7(63.6 \%)$ & $30(33 \%)$ & \\
\hline Massive infarction & $3(27.2 \%)$ & 35 (48.4\%) & \\
\hline Multiple infarction & $1(9 \%)$ & $16(17.4 \%)$ & \\
\hline Old infarctions & $1(9 \%)$ & $1(1 \%)$ & \\
\hline $\begin{array}{l}\text { White matter } \\
\text { ischemia }\end{array}$ & $2(18.2 \%)$ & $1(1 \%)$ & \\
\hline NIHSS & $15.2 \pm 4.7$ & $15.2 \pm 4.4$ & 0.886 \\
\hline
\end{tabular}

MV morphological variations, ICA internal carotid artery, TIA transient ischemic attack, CVS cerebrovascular stroke, NIHSS National Institute of Health Stroke Scale

which was different from our findings. Unlike our results, they observed statistically significant MV in females $>60$ years, while there was no sex difference in patients $<60$ years. Their patients' MV were bilateral in CA in 48\%; in the remaining patients, the MV of the left side were common than those of the right like our results. They noticed that MV have no significance as a risk factor for stroke or any cerebrovascular disease.

Martins and colleagues [7] studied 19,804 patients with multiple risk factors not including IS (D.M., AF, hypertension, and hyperlipidemia). They used carotid ultrasound and detected MV of the ICA among $13.52 \%$ among their patient, which is slightly higher than our prevalence. Kinking followed by coiling was the most common MV detected by them which was not in concordance with our results. The prevalence of MV was higher among older age and female patients and was in the left ICA in most patients. Again, their results were in concordance with the results of our study. Contrary to our results, they observed that only kinking of ICA was significantly correlated with IS. Moreover, they found significant hemodynamic disorders in the ICA associated with kinking, which related to the occurrence of IS. The differences between their findings and ours could be attributed to their large sample size and the small number of patients with kinking examined by us.

Our results were higher than the rate recorded by YiSin study [6] who used carotid ultrasound to study the prevalence of ICA MV among 615 patients with various risk factors (D.M., hypertension, IS, and hyperlipidemia). He found that the prevalence among the general population was $4.1 \%$ and the prevalence among IS patients was $4.7 \%$ only. The big differences between our MV prevalence and his prevalence could be attributed to the different ethnicity as the Asian population has intracranial vascular changes common than extracranial vascular changes $[18,19]$. He found also that ICA MV are not related to IS occurrence and stroke severity, which was like our findings. From previous results, we could conclude that the prevalence of MV among the Egyptian population lies midway between high prevalence among Caucasians and low prevalence among the Asian population.

Beigelman et al. [20] examined 60 consecutive stroke patients with carotid kinking using carotid ultrasonography and found no differences between flow velocity assessed at the level of kinking compared to the normal vessel. They concluded that carotid kinks are not a mechanism of acute cerebral ischemia which was similar to our results.

Sang Mi-Noh and Kang [21] examined 27 stroke patients using CTA to examine the relation between carotid artery morphology and the occurrence of IS, and they found that ICA MV has a significant impact on the IS occurrence. These positive results could be attributed to the use of CTA in their research which made them detect more number of MV in addition to their small sample size which made positive relation more likely.

\section{Conclusion}

Morphological variations in the course of the internal carotid artery are frequently observed during carotid ultrasound study. According to our study, their presence was associated with aging, female gender, and vascular risk factors as diabetes and hypertension. The results of this study also indicate that MV are not associated with an increased risk of ischemic stroke. Further large population-based studies are encouraged to estimate whether MV are associated with an increased risk of CVS or not. 


\section{Abbreviations}

MV: Morphological variations; ICA: Internal carotid artery; TIA: Transient ischemic attack; CVS: Cerebrovascular stroke; NIHSS: National Institute of Health Stroke Scale; D.M.: Diabetes mellitus; HTN: Hypertension; IS: Ischemic stroke

\section{Acknowledgements}

Not applicable

\section{Authors' contributions}

NA: supervisor and data analysis. HA: acquisition of data and study design MN: acquisition of data and data analysis AN: acquisition of data, study design, and editing of the manuscript The authors read and approved the final manuscript.

\section{Funding}

Not applicable (no funding received in this study)

\section{Availability of data and materials}

The datasets used and/or analyzed during the current study are available from the corresponding author on reasonable request.

\section{Ethics approval and consent to participate}

This study protocol was approved by the ethical committee at the Faculty of Medicine, Assuit University, in October 2016 with IBR no. 17101047. All participant patients or their 1st degree of relatives were giving informed consent for the participation in the study before assessment. All patients' data were confidentially kept.

\section{Consent for publication}

Not applicable

\section{Competing interests}

The authors declare that they have no competing interests in this section.

\section{Author details}

${ }^{1}$ Neurology Department, Assuit University, Assiut 71526, Egypt. ${ }^{2}$ Vascular and Endovascular Surgery Department, Assiut University, Assiut, Egypt.

Received: 28 May 2020 Accepted: 6 August 2020

Published online: 28 August 2020

\section{References}

1. Abd-Allah F, Khedr E, Oraby M, Bedair A, Georgy S, Moustafa R. Stroke burden in Egypt: data from five epidemiological studies. J Neurosci. 2017; 128:1-16.

2. Khedr EM, Fawi G, Abdela M, Mohammed TA, Ahmed MA, El-Fetoh NA, et al. Prevalence of ischemic and hemorrhagic strokes in Qena Governorate, Egypt: community-based study. J Stroke Cerebrovasc Dis. 2014;23(7):1843-8.

3. Benamer HT, Grosset D. Stroke in Arab countries: a systematic literature review. J Neurol Sci. 2009;284(1-2):18-23.

4. Weibel J, Fields WS. Tortuosity, coiling, and kinking of the internal carotid artery. II Relationship of morphological variation to cerebrovascular insufficiency. 1965;15(5):462-.

5. Alsini AY, Ibrahim A. Pulsating tonsil due to medial displacement of the internal carotid artery. Am J Case Rep. 2017;18:502-6.

6. Wong Y-S, Ong C-T, Sung S-F, Wu C-S, Hsu Y-C, Su Y-H, et al. Association between abnormal course of carotid artery and cerebrovascular disease. Acta Neurol Taiw. 2014;23:90-4.

7. Martins HFG, Mayer A, Batista P, Soares F, Almeida V, Pedro AJ, et al. Morphological changes of the internal carotid artery: prevalence and characteristics. A clinical and ultrasonographic study in a series of 19804 patients over 25 years old. Eur J Neurol. 2018;25(1):171-7.

8. Radak D, Babic S, Tanaskovic S, Matić P, Sotirovic V, Stevanovic P, et al. Are the carotid kinking and coiling underestimated entities? Vojnosanit Pregl. 2012;69:616-9.

9. Macchi C, Gulisano M, Giannelli F, Catini C, Pratesi C, Pacini P. Kinking of the human internal carotid artery: a statistical study in 100 healthy subjects by echocolor Doppler. J Cardiovasc Surg (Torino). 1997;38(6):629-37.
10. Pancera P, Ribul M, Presciuttini B, Lechi A. Prevalence of carotid artery kinking in 590 consecutive subjects evaluated by Echocolordoppler. Is there a correlation with arterial hypertension? Arch Intern Med. 2000;248:7-12.

11. Paulsen F, Tillmann B, Christofides C, Richter W, Koebke J. Curving and looping of the internal carotid artery in relation to the pharynx: frequency, embryology and clinical implications. J Anat. 2000;197 Pt 3(Pt 3):373-381.

12. Grant EG, Benson CB, Moneta GL, Alexandrov AV, Baker JD, Bluth El, et al. Carotid artery stenosis: gray-scale and Doppler US diagnosis--Society of Radiologists in Ultrasound Consensus Conference. J Radiol. 2003;229(2): 340-6.

13. Togay-lsikay C, Kim J, Betterman K, Andrews C, Meads D, Tesh P, et al. Carotid artery tortuosity, kinking, coiling: stroke risk factor, marker, or curiosity? Acta Neurol Belg. 2005;105(2):68.

14. Vannix RS, Joergenson EJ, Carter R. Kinking of the internal carotid artery. Clinical significance and surgical management. Am J Surg. 1977:134(1):82-9.

15. Saba L, Argiolas GM, Sumer S, Siotto P, Raz E, Sanfilippo R, et al. Association between internal carotid artery dissection and arterial tortuosity. J Neuroradiol. 2015;57(2):149-53.

16. Baracchini C, Farina F, Tonello S, Citton V, Meneghetti G, Ballotta E, et al. Endothelial dysfunction in carotid elongation. J Neuroimaging. 2013;23(1): $18-20$.

17. Sacco S, Totaro R, Baldassarre M, Carolei A. Morphological variations of the internal carotid artery: prevalence, characteristics and association with cerebrovascular disease. Int J Angiol. 2007;16(2):59-61.

18. Hua $Y$, Jia $L$, Xing $Y$, Hui $P$, Meng $X$, Yu D, et al. Distribution pattern of atherosclerotic stenosis in Chinese patients with stroke: a multicenter registry study. Aging Dis. 2019;10(1):62-70.

19. Sacco RL, Kargman DE, Gu Q, Zamanillo MC. Race-ethnicity and determinants of intracranial atherosclerotic cerebral infarction. NOMAS. Stroke. 1995;26(1):14-20.

20. Beigelman R, Izaguirre A, Robles M, Grana D, Ambrosio G, Milei J. Kinking of carotid arteries is not a mechanism of cerebral ischemia: a functional evaluation by Doppler echography. Int Angiol. 2011;30(4):342-8.

21. Noh SM, Kang HG. Clinical significance of the internal carotid artery angle in ischemic stroke. Sci Rep. 2019:9(1):4618.

\section{Publisher's Note}

Springer Nature remains neutral with regard to jurisdictional claims in published maps and institutional affiliations.

\section{Submit your manuscript to a SpringerOpen ${ }^{\circ}$ journal and benefit from:}

- Convenient online submission

- Rigorous peer review

- Open access: articles freely available online

High visibility within the field

- Retaining the copyright to your article

Submit your next manuscript at $\boldsymbol{\nabla}$ springeropen.com 